\title{
Smartphone-Based Safety Planning and Self-Monitoring for Suicidal Patients: A Conceptual Basis for the CASPAR (Continuous Assessment for Suicide Prevention and Research) Study
}

\author{
Chani Nuij 1,*, Wouter van Ballegooijen 1,2, Jeroen Ruwaard 1, Derek de Beurs 1,3, \\ Rory C. O'Connor ${ }^{4}$, Jan H. Smit ${ }^{2}$, Heleen Riper ${ }^{1}$ and Ad Kerkhof ${ }^{1}$ \\ 1 Section Clinical Psychology, Vrije Universiteit Amsterdam, Van der Boechorststraat 1, \\ 1081 BT Amsterdam, The Netherlands; w.van.ballegooijen@vu.nl (W.v.B.); j.j.ruwaard@vu.nl (J.R.); \\ d.debeurs@nivel.nl (D.d.B.); h.riper@vu.nl (H.R.); a.j.f.m.kerkhof@vu.nl (A.K.) \\ 2 GGZ inGeest, Amsterdam, The Netherlands; jh.smit@ggzingeest.nl \\ 3 Netherlands Institute for Health Services Research (Nivel), 3513 CR Utrecht, The Netherlands \\ 4 Institute of Health and Wellbeing, University of Glasgow, Glasgow G12 8QQ, UK; \\ Rory.OConnor@glasgow.ac.uk \\ * Correspondence: c.nuij@vu.nl
}

\begin{abstract}
Suicidal behaviour remains difficult to predict and prevent, even for experienced mental health care professionals. The known distal risk factors for suicidal behaviour are not sufficiently specific to fully understand the complex dynamic processes that precede a suicide attempt. Realtime mobile monitoring data can be used to analyse proximal risk mechanisms within the suicidal process. At the same time smartphone-based safety planning and self-monitoring may enhance a patient's self-management skills thereby increasing their capacity to respond to a suicidal crisis and to become more aware of crisis symptoms. The current paper describes the theoretical and conceptual rationale for the CASPAR study which applies an innovative approach to the study of suicidal processes. It uses basic science approaches to inform the implementation of an innovative suicide prevention intervention. We aim to develop and implement mobile safety plan in conjunction with real-time monitoring in order to both directly implement suicide prevention interventions and to study the ongoing dynamics of individual suicidal behaviour by applying network analysis.
\end{abstract}

Keywords: suicide prevention; e-mental health; implementation; fundamental research; ecological momentary assessment; experience sampling; network analysis

\section{Introduction}

Suicidal behaviour results from a complex interaction between biological, social and psychological factors (e.g., gender, the experience of stressful life events and mental disorders) [1,2]. Our understanding of the factors associated with suicide has grown in recent decades, but it is still unclear how those factors interact with each other [2]. As a consequence of this indistinctness, suicidal risk factors are of limited clinical use in the identification of individuals who are most at risk of acting on their thoughts of suicide $[1,3,4]$. Even for experienced mental health care professionals suicidal behaviour remains extremely difficult to predict and prevent [4,5]. According to a review of 40 studies, approximately one-third of people who die by suicide had been in contact with mental health care in the year before their death, and about one in five had contact with a professional in the month before death [6]. Unfortunately, there is no gold-standard treatment for prevention of suicidality [7]. To improve prevention and treatment strategies, the field of suicidology would benefit from new insights into the psychopathology of suicidal processes. 
In this paper, we describe the theoretical and conceptual rationale for the CASPAR study (Continuous Assessment for Suicide Prevention and Research). It will apply a novel approach to investigate suicidal processes, by combining implementation of a smartphone-based intervention for safety planning with basic science on suicidal processes. We will implement two mobile health applications to complement mental health care for suicidal patients in treatment, with the aim of improving their self-management skills to cope with a suicidal crisis and help the patient increase their awareness of crisis symptoms. A total of 60 participants will be recruited from three mental health care institutes in the Netherlands (e.g. GGZ InGeest, Parnassia and GGZ Delfland). We will investigate the dynamics of suicidal processes by using the data we will obtain from the implementation of these applications.

\section{Safety planning}

\subsection{Safety planning approach}

Safety planning is a psychosocial intervention used in treatment of high-risk suicidal patients (presence of significant suicidal ideation, suicidal intent or/and a recent suicide attempt) [8]. The basic premise underpinning the safety plan approach is that it increases patient's self-management competencies when faced with a suicidal crisis. It is a collaborative process between patient and clinician, win which potential coping strategies (e.g., what patients can do when they are in an acute suicidal crisis) are planned [9]. Safety planning strategies are used widely, for example, they form part of crisis response plans (e.g., Rudd et al. [10]) and are embedded in multiple treatments targeting suicidal behaviour like the collaborative assessment and management of suicidality (CAMS) [11] and cognitive-behavioural therapy for suicide prevention (CBT-SP) [12]. More recently, Stanley and Brown [13] have developed a specific safety planning tool. Their safety plan consists of a written, prioritised list of warning signs, personalized coping strategies and sources of support that patients can use to alleviate a suicidal crisis. Stanley and Brown's safety planning tool is a systematic and comprehensive approach to maintaining safety in suicidal patients [13].

Little research has studied the effects of safety planning on suicidal behaviour [14], but attitudes of both patients and clinicians towards safety planning has been positive $[12,15]$. With a safety plan, the patient is neither helpless nor alone [14]. Dutch suicide prevention guidelines recommend this intervention alongside treatment as usual (TAU) for at-risk mental health care patients [16]. However, safety planning is often not implemented in practice [17], possibly due to the limited feasibility of traditional written safety plans which are either carried around or stored at the patients' home. Paper safety plans may not always be at hand in times of crisis, which is crucial considering the fluid nature of suicidal thinking and behaviour [9].

\subsection{Safety planning app: MYPLAN}

Mobile technology provides new opportunities for personalised mental health care. Customized programs, called applications (apps), can be run on mobile devices for support, coaching or gathering information [18]. The World Health Organization (WHO) has recommended mobile devices as an option for providing support and therapy to people at risk of suicide [19]. Apps can be especially useful for suicide prevention interventions, because of their ability to deliver support and interventions in situ and at the time of crisis, as suicide ideation and behaviour can change rapidly [20].

Most available apps targeting suicidal behaviour have limited interactivity and provide little more than a direct link to local or national support hotlines [21], for example the app 'HELP Prevent Suicide' for Android and iOS (https://appadvice.com/game/app/help-prevent-suicide/791283546). One exception is MYPLAN, which is an interactive self-help smartphone app developed by Larsen (http://www.minplan.org), based on Stanley and Brown's safety plan intervention [13]. With interactive menus, audio and video, MYPLAN allows for more dynamic, personalised and engaging safety plans compared to the traditional paper-and-pencil methods and other apps targeting suicidal 
behaviour (see Figure 1). MYPLAN facilitates dealing with a suicidal crisis by improving recognition of crisis symptoms and establishing the user's own experience with self-help skills.

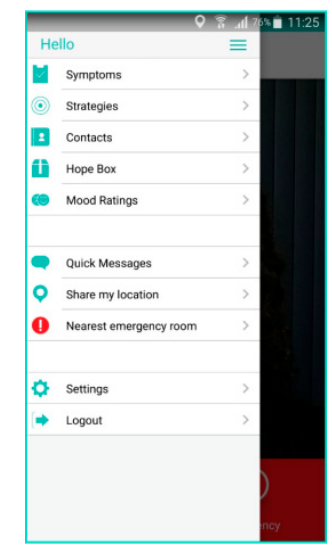

(a)

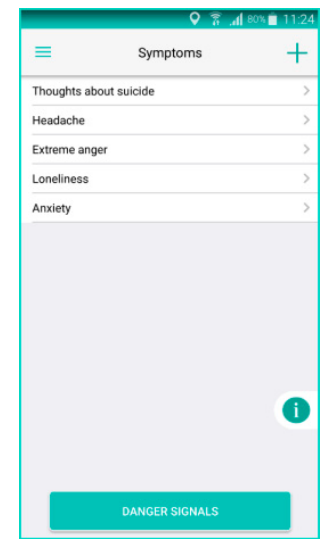

(b) (c)

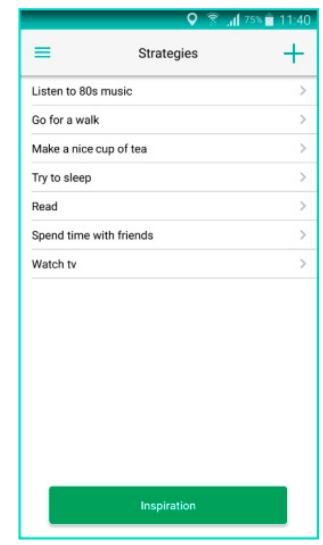

(d)

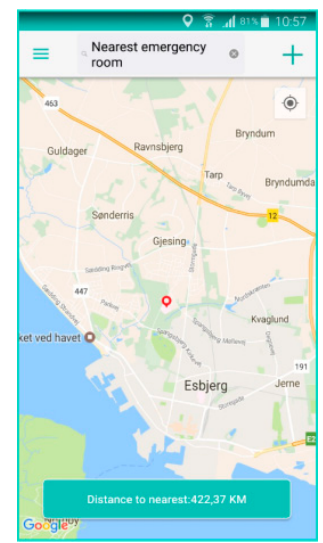

Figure 1. Screenshots of MYPLAN: (a) Menu; (b) Symptoms; (c) Strategies; (d) Nearest emergency room.

MYPLAN consist provide several sections:

- Symptoms (Figure 1.b), where personal signs of a pending crisis can be noted

- Strategies (Figure 1.c), where personal coping strategies for crisis situations can be added

- Contacts, where contact persons can be included

- Hope Box, where personal images, music or web links can be added to recall reasons for living

- Mood ratings, to assess the severity of suicidal thoughts and to monitor mood

- Quick Messages, where predefined messages can be sent in a crisis

- Share my location, through which patients can share their location with contacts

- Nearest emergency room (Figure 1.d), which helps the user locate the nearest psychiatric emergency service

The feasibility of MYPLAN has been explored in a study conducted in Denmark and Norway [22]. In this study, patients responded positively to MYPLAN. In general they said that they experienced more ownership of their crisis strategies and actually used the app in times of crisis. MYPLAN is currently being investigated in Denmark in a randomised controlled trail (RCT) for its effectiveness on people at risk of suicide compared to the paper version of the safety plan, with suicide ideation as primary outcome measure (https://clinicaltrials.gov/ct2/show/NCT02877316). The CASPAR study runs parallel to this study and focuses on implementation of MYPLAN alongside basic science.

\subsection{MYPLAN in the CASPAR study}

In the CASPAR study, we are cooperating with the developers of MYPLAN to translate the safety planning app into Dutch and to implement the app in three mental health care institutes $(n=60)$ in the Netherlands. To further encourage a patient's self-management outside treatment hours, we will ask patients to install a self-monitoring app that tracks mental health variables which will run alongside MYPLAN. 


\section{Real-time self-monitoring}

\subsection{Ecological Momentary Assessment}

Self-monitoring techniques such as experience sampling or Ecological Momentary Assessment (EMA) [23] have been used to monitor psychiatric processes by daily repeated self-report questions [24]. The repeated assessments monitor behaviour and experiences over time and across settings, hereby enhancing ecological validity of questionnaires compared to traditional retrospective questionnaires. Furthermore, EMA reduces the likelihood of retrospection and recall biases that are associated with traditional questionnaires [24].

Early versions of EMA relied on personal diaries with paper questionnaires that participants had to fill in at various times throughout the day prompted by watches or beepers [25]. Technological advances have made it possible to use electronic questionnaires instead of paper-based diaries. Handheld computers send prompts by themselves, hereby eliminating the back-filling problem (i.e., the possibility that participants may not respond to the questionnaires at the appropriate time or complete them all at once) that is associated with paper-based EMA [26]. Disadvantages of hand-held computers, like the necessity for participants to be trained in using the device and the need to carry around an extra device during the study, can be avoided by the use of EMA on personal smartphones [27]. Conducting EMA on personal smartphones with a major depressive disorder has already been shown to be feasible and low-cost [28].

EMA is often used as a method of measuring dynamic processes, but some studies have reported behavioural changes in participants over the EMA monitoring period, thereby suggesting that ambulatory monitoring may constitute a therapeutic intervention itself [29]. EMA studies on substance dependence [30,31] and chronic pain [32] suggest that intensive repeated assessment draws attention to the monitored behaviour and psychological states for which patients may otherwise have been inattentive. Insights into the nature and dynamics of the patients own symptomatology may engender feelings of control and empowerment in relation to their symptoms [29].

\subsection{Ecological Momentary Assessment with suicidal patients}

Two studies have shown the feasibility of electronic monitoring of suicidal ideation, using handheld computers [33,34]. Nock et al. [33] used EMA to measure suicidal and non-suicidal self-injurious thoughts and behaviours as they occurred in real-time, demonstrating the feasibility of using EMA in this group. In Husky et al.'s study [34] the feasibility of EMA in four samples at varying risk for suicide (e.g., recent suicide attempters, past suicide attempters, affective controls and healthy controls) was determined. Results indicated that repeated assessment by EMA variables assessing mood, suicidal ideation and self-harm with participants at risk for suicide is feasible, with the latter showing high compliance and no adverse effects. Importantly, the endorsement of negative thoughts and suicidal ideation were not reactive to the repeated measurements. These results are consistent with reports indicating that urges to self-harm do not increase after the assessment of suicidal ideation with retrospective questionnaires [35]. Husky et al. [34] suggest that ambulatory monitoring may even have an interventional effect on participants at risk for suicide. Possible positive reactive effects of EMA, defined as a decrease in hopelessness and an increase in activities, were only found in the high risk group after self-monitoring for one week.

\subsection{Ecological Momentary Assessment in CASPAR}

In the CASPAR study we aim to develop and implement our own EMA app for smartphones (both for iOS and Android operating systems) to enhance self-management of suicidal patients. The EMA app will be implemented into three mental health institutes in the Netherlands, together with MYPLAN. Each patient that participates in the CASPAR study will have two apps on their smartphones. 
The EMA assessments in CASPAR will be direct, e.g. prompted single-item self-report questions, and indirect, e.g. unobtrusively collected variables that accumulate silently on the smartphone of patients without their interference, for example GPS-data, physical activity and the use of social apps by the patient. Those proxies of mental health measured by unobtrusive EMA (e.g. behavioural patterns, contextual triggers and social activity) are assumed to be clinically useful [36,37]. For example, in an exploratory study using mobile phone sensor data, behavioural markers were related to depressive symptom severity [37]. Unfortunately, unobtrusive EMA is a young method and the true potential has still to be unlocked [38].

For the direct assessments, we will create EMA items based on existing validated questionnaires related to suicidology, like the Patient Health Questionnaire (PHQ) [39] and the Short Defeat and Entrapment Scale (SDES) [40], to enable self-monitoring. An example of an EMA-based question is 'In the past hour I was feeling down'. The respondent can answer their level of agreement on a 7point Likert scale ranging from disagree (-3) to agree (3).

The patient will have access to their own EMA results. The course of each variable over time (of both direct and indirect assessment) will be accessible in the app, displayed in user friendly graphics. These results give the patient insight into the dynamic fluctuations and connections between their own symptomatology and daily life patterns hereby enhancing their feelings of control and empowerment in relation to their symptoms. To carry out self-monitoring and to use the EMA results in treatment will require the active engagement of the patient in their own recovery, thereby enhancing their responsibility of progress and self-management [29].

In future, the EMA data may be used in therapeutic interventions. Patients and clinicians could discuss the past week by looking at the monitoring data displayed on the patient's smartphone. This will reduce the recall bias of patients because they do not have to rely only on their memory. The clinician will get a more complete view of the patient's changes in symptoms, which will improve the understanding of the therapeutic processes as well as mental processes and suicidal ideation and behaviour of the patients over time [29,41].

In addition to potential clinical benefits, EMA data can be used for basic science. Many investigations of suicidal processes focus on bivariate associations between theoretical demographic or psychiatric factors and suicidal behaviours [42] even though there is no debate among epidemiologists that suicidal behaviours are complex and determined by multiple phenomena $[1,4]$. The CASPAR study aims to use EMA data to monitor the multidimensional unfolding of behavioural and emotional processes in real-time. With the data we hope to capture the dynamics of suicidal processes of individual patients better [26].

\section{Dynamic model of suicidal processes}

With regard to the multiple risk factors, the Integrated Motivational-Volitional (IMV) model of suicidal behaviour was developed by O'Connor [43] to conceptualise suicidality. This model will be used in the CASPAR study as a framework to study suicidal processes.

\subsection{Integrated Motivational-Volitional (IMV) model of suicidal behaviour}

The IMV model integrates key factors from earlier theories, like the Theory of Planned Behaviour [44], the diathesis-stress hypothesis [45] and the arrested flight model of suicidal behaviour [46], to map the transition from suicidal thoughts to suicidal behaviour (Figure 2). In the IMV model, suicidal behaviour is conceptualised as a behaviour that develops through motivational and volitional phases. The motivational phase describes the factors that govern the development of suicidal ideation and intent, e.g. defeat, worrying and entrapment and rumination. The volitional phase factors govern the transition from suicidal thinking to suicidal behaviour and include access to the means of suicide, the personal capacity for carrying out suicide and impulsivity [1,43] 


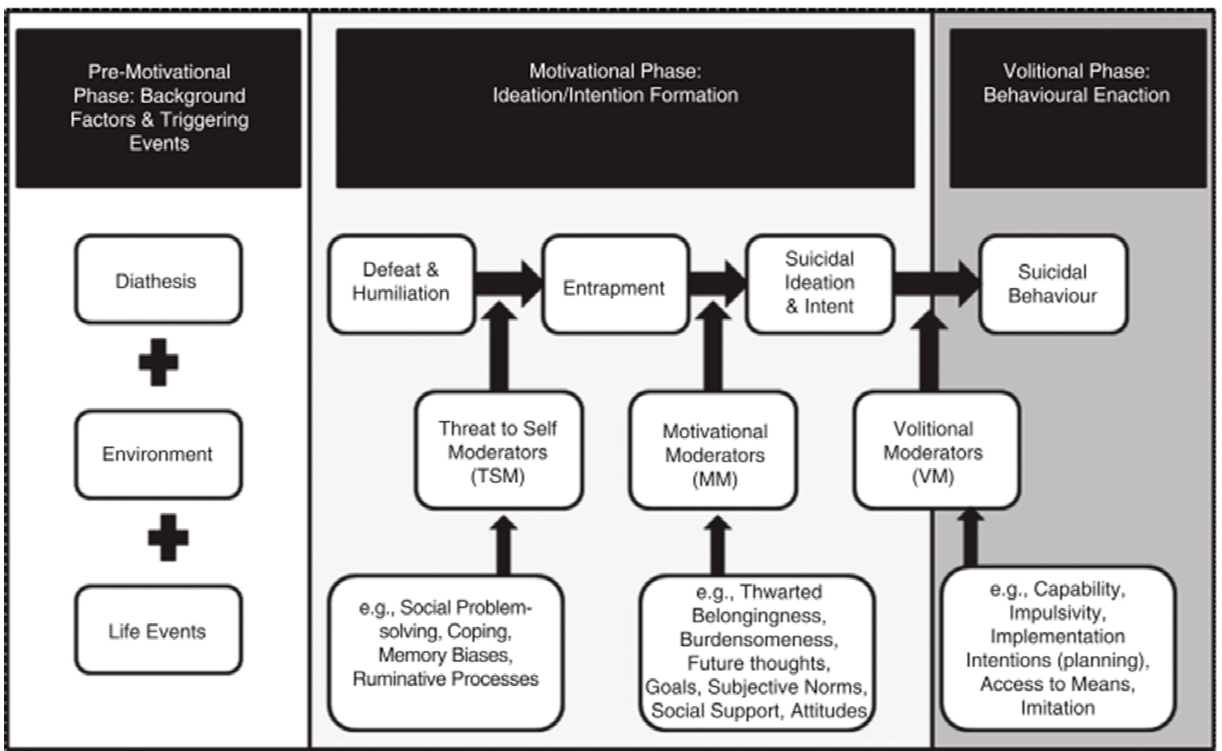

Figure 2. Integrated Motivational-Volitional (IMV) Model of Suicidal Behaviour; from O'Connor 2011 [43].

There is growing evidence in supports of the IMV model with several studies having yielded support for the central tenets of the model. Higher levels of defeat are associated with suicide ideation [47] and entrapment, defined as blocked attempts to escape from high stress or defeating circumstances, was found to be the most important modifiable predictor of repeat suicidal behaviour four years after a previous attempt [48]. Other studies have demonstrated that volitional phase factors are key to the emergence of suicidal behaviour, like impulsivity [49] and access to means [50,51]. As these studies are based on retrospectively collected data they may lack ecological validity [52]. Validation of the postulated transition from one stage to the next requires assessment of day-to-day fluctuations and patterns in the burden, intensity, severity and duration of suicidal ideation, rumination and other relevant factors, as outlined within the IMV model [43].

\subsection{IMV model and analysis in the CASPAR study}

The CASPAR study aims to receive real-time data with EMA, both from the direct and indirect assessments. With these data we hope to capture the dynamics of psychological processes and stages of suicidal processes, both on an aggregated and individual level. To understand suicidal processes, multiple psychometric techniques will be used.

For each individual patient, we will construct longitudinal pathways by using latent class mixed modelling. Path analysis (as a structural equation model, e.g. Bagozzi \& Yi, 2012 [53]) will be used to validate the expected coherence of factors based on the IMV model. To grasp the complex organization of multiple symptoms we will use the network approach. This approach conceptualizes symptoms as mutually interacting, often reciprocally reinforcing, elements of a complex network [54,55]. Network analysis have been successfully used in the field of psychosis [56], personality research [57] and depression [58], contributing to advances in psychopathology research. For instance, non-DSM symptoms of depression (e.g. sympathetic arousal, somatic complaints and panic) [59] were found to be equivalent connected with other symptoms in a network as DSM depression symptoms [60]. The network approach has yielded promising results in many different sectors and first steps of using this approach in the field of suicidology are made [61]. The CASPAR study will take the next step by using the network approach based on EMA data in this population.

\section{Discussion}

Suicidal behaviour is difficult to predict and prevent, even for experienced mental health care professionals. The known risk factors for suicidal behaviour are often static and distal, and researched in bivariate associations, which are not sufficiently specific to describe the complex dynamic 
processes of suicidality. More sophisticated techniques are required to enhance existing theories and to improve treatment and prevention of suicidal behaviours. At the moment there are several recommended evidence based treatments for suicidality, and in all of these treatments an important intervention is safety planning [16]. Identifying coping strategies and defining sources of support for patients for use in times of crisis helps those who are vulnerable to better recognise symptoms and develop self-help skills. Though, the safety plan intervention is often not implemented in practices [17], possible due to the limited feasibility of traditional written form. To this end, the electronic suicide safety app MYPLAN was developed to increase the availability of care at times of crisis compared to the traditional paper-based plans, hereby extending care for suicidal patients from one hour a week in a private session to 24/7. Another tool for enhancing self-management of patients is self-monitoring. Intensive repeated assessment can draw attention to the monitored behaviour and psychological states. Awareness of the patients' own symptomatology may engender feelings of control in relation to their symptoms.

The CASPAR study aims to implement MYPLAN and an EMA app for real-time self-monitoring in three mental health care institutes in the Netherlands $(n=60)$ to enhance self-management of patients at risk of suicide. Patients will have access to their own EMA results, displaying the course of each variable over time in graphics. These results give the patient an insight into the dynamic fluctuations and inter-connections between their own symptomatology and daily life patterns, hereby enhancing their feelings of control and empowerment. The EMA data will also be used in therapy, when the patient shares his or her data. The clinician will get a more complete view of the patient's changes in symptoms, which will improve understanding of the individual therapeutic process. The monitoring data can also give an indication of the effect of interventions, allowing professionals and patients to change course if the desired effect is not achieved. Clinicians can specify interventions based on the needs of the individual patient. Of course, to achieve the goals of selfmonitoring and to successfully use the EMA results in treatment will require active engagement of the patient in their recovery, thereby enhancing their responsibility of progress and self-management. Feasibility of the implementation will be tested by usability (e.g. the extent to which the applications are easy to use and accessible) [62,63], uptake and acceptability of using both tools and at last the acceptability of data sharing by the patients. Due to the novelty of this project, outcomes of implementation do not have a pre-defined endpoint but will be closely monitored.

A second aim of the CASPAR study is to use the EMA data to conduct basic science research into the psychological processes that underpin suicidal ideation and behaviour. Specifically, we will use the data to empirically test the hypothesised psychological processes and pathways as outlined within the IMV model of suicidal behaviour. Finally, CASPAR aims to identify individual pathways to suicidal behaviour identify specific profiles of subtypes of suicidal individuals.

After ending the CASPAR study, we will further implement the safety plan and self-monitoring tools in mental health care and beyond. The results of fundamental research will inform guidelines and treatment development. We will disseminate the results of our fundamental research through several different national and international networks (e.g. 113Online, NIVEL, IASP and ISRII).

Despite these high expectations some points of discussion have to be appointed. The CASPAR study will include chronic depressive participants at risk of suicide. With life and dead at stake, this group of participants is vulnerable [35]. Their vulnerability can lead to questions of competence and voluntariness of suicidal patients to participate in research, often resulting in more ethical concerns regarding methodologies and research protocols than studies of more innocuous issues. For example, the potential negative influence on a suicidal patient when assigned to a non-treatment control group [64]. The CASPAR study will apply a new method to study suicidology in which we will complement current treatments to enhance self-management in effort to gain data to study basic science. An expected consequence of this method is a mutual influence of MYPLAN and EMA. We expect that the use of MYPLAN will have effect on the results of EMA data and we assume that EMA will influence the participants as well. Thereby, we will study chronic depressive patients at risk of suicide who are in treatment. According to us a new method to study this high-risk group in an ethical way. 
In sum, this project should lead to better self-management of suicidal patients and simultaneously studies of the dynamics of suicidal processes, potentially resulting in less suicidal behaviour in patients and more insight in the dynamics of suicidal behaviour. The CASPAR project started in the Netherlands on the first of November 2016 and is currently in the preparatory phase. The start of implementation is planned in 2018.

Acknowledgments: This study is funded by ZonMW, project number 537001008.

Conflicts of Interest: The authors declare no conflict of interest.

\section{References}

1. O'Connor, R. C.; Nock, M. K. The psychology of suicidal behaviour. The Lancet Psychiatry 2014, 1, 7385.

2. Franklin, J. C.; Ribeiro, J. D.; Fox, K. R.; Bentley, K. H.; Kleiman, E. M.; Huang, X.; Musacchio, K. M.; Jaroszewski, A. C.; Chang, B. P.; Nock, M. K. Risk Factors for Suicidal Thoughts and Behaviors: A Meta-Analysis of 50 Years of Research. Psychol. Bull. 2016, 143, 187-232.

3. Ribeiro, J. D.; Joiner, T. E. The interpersonal-psychological theory of suicidal behavior: current status and future directions. J. Clin. Psychol. 2009, 65, 1291-1299.

4. Nock, M. K.; Borges, G.; Bromet, E. J.; Cha, C. B.; Kessler, R. C.; Lee, S. Suicide and Suicidal Behavior. Epidemiol. Rev. 2008, 30, 133-154.

5. Gysin-Maillart, A.; Schwab, S.; Soravia, L.; Megert, M.; Michel, K. A Novel Brief Therapy for Patients Who Attempt Suicide: A 24-months Follow-Up Randomized Controlled Study of the Attempted Suicide Short Intervention Program (ASSIP). PLoS Med. 2016, 13, e1001968.

6. Luoma, J. B.; Martin, C. E.; Pearson, J. L. Contact With Mental Health and Primary Care Providers Before Suicide: A Review of the Evidence. Am. J. Psychiatry 2002, 159, 909-916.

7. Klonsky, E. D.; May, A. M.; Saffer, B. Y. Suicide, Suicide Attempts, and Suicidal Ideation. Annu. Rev. Clin. Psychol. 2016, 12, 307-330.

8. Pearson, J. L.; Stanley, B.; King, C. A.; Fisher, C. B. Intervention research with persons at high risk for suicidality: safety and ethical considerations. J. Clin. Psychiatry 2001, 62, 17-26.

9. Kennard, B. D.; Biernesser, C.; Wolfe, K. L.; Foxwell, A. A.; Lee, S. J. C.; Rial, K. V; Patel, S.; Cheng, C.; Goldstein, T.; Mcmakin, D. Developing a brief suicide prevention intervention and mobile phone application : a qualitative report. J. Technol. Hum. Serv. 2015, 33, 345-357.

10. Rudd, M. D.; Joiner, T.; Rajab, M. H. Treating suicidal behavior: an effective, time-limited approach; The Guildford Press: New York, 2001.

11. Jobes, D. A. Managing suicidal risk: a collaborative approch; The Guildford Press: New York, 2006.

12. Stanley, B.; Brown, G.; Brent, D. A.; Wells, K.; Poling, K.; Curry, J.; Kennard, B. D.; Wagner, A.; Cwik, M. F.; Klomek, A. B.; Goldstein, T.; Vitiello, B.; Barnett, S.; Daniel, S.; Hughes, J. Cognitive-behavioral therapy for suicide prevention (CBT-SP): treatment model, feasibility, and acceptability. J. Am. Acad. Child Adolesc. Psychiatry 2009, 48, 1005-1013.

13. Stanley, B.; Brown, G. K. Safety Planning Intervention: A Brief Intervention to Mitigate Suicide Risk. Cogn. Behav. Pract. 2012, 19, 256-264.

14. Kayman, D. J.; Goldstein, M. F.; Wilsnack, J.; Goodman, M. Safety Planning for Suicide Prevention. Curr. Treat. Options Psychiatry 2016, 3, 411-420.

15. Brown, G. K.; Ten Have, T.; Henriques, G. R.; Xie, S. X.; Hollander, J. E.; Beck, A. T. Cognitive therapy for the prevention of suicide attempts: a randomized controlled trial. JAMA 2005, 294, 563-570.

16. Van Hemert, A. M.; Kerkhof, A. J. F. M.; De Keijser, J.; Verwey, B.; Van Boven, C.; Hummelen, J. W.; De Groot, M. H.; Lucassen, P.; Meerdinkveldboom, J.; Steendam, M.; Stringer, B.; Verlinde, A. A.; van de Glind, G. Multidisciplinaire richtlijn diagnostiek en behandeling van suïcidaal gedrag; 2012.

17. de Beurs, D. P.; de Groot, M. H.; de Keijser, J.; Mokkenstorm, J.; van Duijn, E.; de Winter, R. F. P.; Kerkhof, A. J. F. M. The effect of an e-learning supported Train-the-Trainer programme on implementation of suicide guidelines in mental health care. J. Affect. Disord. 2015, 175, 446-453.

18. Torous, J.; Roberts, L. W. The Ethical Use of Mobile Health Technology in Clinical Psychiatry. J. Nerv. Ment. Dis. 2017, 205, 4-8.

19. World Health Organization Preventing suicide: a global imperative; 2014. 
20. Larsen, M. E.; Nicholas, J.; Christensen, H. A Systematic Assessment of Smartphone Tools for Suicide Prevention. PLoS One 2016, 11, e0152285.

21. Aguirre, R. T. P.; McCoy, M. K.; Roan, M. Development Guidelines from a Study of Suicide Prevention Mobile Applications (Apps). J. Technol. Hum. Serv. 2013, 31, 269-293.

22. Larsen, J. L. S.; Frandsen, H.; Erlangsen, A. MYPLAN - A mobile phone application for supporting people at risk of suicide. Crisis 2016, 37, 236-240.

23. Stone, A. A.; Shiffman, S. Ecological momentary assessment (EMA) in behavioral medicine. Ann. Behav. Med. 1994, 16, 199-202.

24. Shiffman, S.; Stone, A. A.; Hufford, M. R. Ecological momentary assessment. Annu. Rev. Clin. Psychol. 2008, 4, 1-32.

25. Bolger, N.; Davis, A.; Rafaeli, E. Diary methods: capturing life as it is lived. Annu. Rev. Psychol. 2003, 54, 579-616.

26. Trull, T. J.; Ebner-Priemer, U. W. Using experience sampling methods/ecological momentary assessment (ESM/EMA) in clinical assessment and clinical research: Introduction to the special section. Psychol. Assess. 2009, 21, 457-462.

27. Courvoisier, D. S.; Eid, M.; Lischetzke, T.; Schreiber, W. H. Psychometric properties of a computerized mobile phone method for assessing mood in daily life. Emotion 2010, 10, 115-124.

28. Torous, J.; Staples, P.; Shanahan, M.; Lin, C.; Peck, P.; Keshavan, M.; Onnela, J.-P. Utilizing a Personal Smartphone Custom App to Assess the Patient Health Questionnaire-9 (PHQ-9) Depressive Symptoms in Patients With Major Depressive Disorder. JMIR Ment. Heal. 2015, 2, e8.

29. Groot, P. C. Patients can diagnose too: How continuous self-assessment aids diagnosis of, and recovery from, depression. J. Ment. Health 2010, 19, 352-362.

30. Freedman, M. J.; Lester, K. M.; McNamara, C.; Milby, J. B.; Schumacher, J. E. Cell phones for ecological momentary assessment with cocaine-addicted homeless patients in treatment. J. Subst. Abuse Treat. 2006, 30, 105-111.

31. Schrimsher, G.; Filtz, K. Assessment Reactivity: Can Assessment of Alcohol Use During Research be an Active Treatment? Alcohol. Treat. Q. 2011, 29, 108-115.

32. Aaron, L. A.; Mancl, L.; Turner, J. A.; Sawchuk, C. N.; Klein, K. M. Reasons for missing interviews in the daily electronic assessment of pain, mood, and stress. Pain 2004, 109, 389-398.

33. Nock, M. K.; Prinstein, M. J.; Sterba, S. K. Revealing the form and function of self-injurious thoughts and behaviors: A real-time ecological assessment study among adolescents and young adults. J. Abnorm. Psychol. 2009, 118, 816-827.

34. Husky, M.; Olié, E.; Guillaume, S.; Genty, C.; Swendsen, J.; Courtet, P. Feasibility and validity of ecological momentary assessment in the investigation of suicide risk. Psychiatry Res. 2014, 220, 564570.

35. Eynan, R.; Bergmans, Y.; Antony, J.; Cutcliffe, J. R.; Harder, H. G.; Ambreen, M.; Balderson, K.; Links, P.S. The effects of suicide ideation assessments on urges to self-harm and suicide. Crisis 2014, 35, 123131.

36. Palmius, N.; Tsanas, A.; Saunders, K. E. A.; Bilderbeck, A. C.; Geddes, J. R.; Goodwin, G. M.; De Vos, M. Detecting Bipolar Depression from Geographic Location Data. IEEE Trans. Biomed. Eng. 2016, 1-1.

37. Saeb, S.; Zhang, M.; Karr, C. J.; Schueller, S. M.; Corden, M. E.; Kording, K. P.; Mohr, D. C. Mobile Phone Sensor Correlates of Depressive Symptom Severity in Daily-Life Behavior: An Exploratory Study. J. Med. Internet Res. 2015, 17, e175.

38. Asselbergs, J.; Ruwaard, J.; Ejdys, M.; Schrader, N.; Sijbrandij, M.; Riper, H. Mobile Phone-Based Unobtrusive Ecological Momentary Assessment of Day-to-Day Mood: An Explorative Study. J. Med. Internet Res. 2016, 18, e72.

39. Kroenke, K.; Spitzer, R. L.; Williams, J. B. The PHQ-9: validity of a brief depression severity measure. J. Gen. Intern. Med. 2001, 16, 606-613.

40. Griffiths, A. W.; Wood, A. M.; Maltby, J.; Taylor, P. J.; Panagioti, M.; Tai, S. The Development of the Short Defeat and Entrapment Scale (SDES). Psychol. Assess. 2015, 27, 1182.

41. Wichers, M.; Simons, C. J. P.; Kramer, I. M. A.; Hartmann, J. A.; Lothmann, C.; Myin-Germeys, I.; van Bemmel, A. L.; Peeters, F.; Delespaul, P.; van Os, J. Momentary assessment technology as a tool to help patients with depression help themselves. Acta Psychiatr. Scand. 2011, 124, 262-272. 
42. Nock, M. K.; Borges, G.; Bromet, E. J.; Cha, C. B.; Kessler, R. C.; Lee, S. Suicide and suicidal behavior. Epidemiol. Rev. 2008, 30, 133-154.

43. O'Connor, R. C. Towards an Integrated Motivational-Volitional Model of Suicidal Behaviour. In International handbook of suicide prevention: Research, policy and practice; O'Connor, R. C.; Platt, S.; Gordon, J., Eds.; Wiley-Blackwell: Chichester, UK, 2011; pp. 181-198.

44. Ajzen, I. The theory of planned behavior. Organ. Behav. Hum. Decis. Process. 1991, 50, 179-211.

45. Schotte, D. E.; Clum, G. A. Problem-solving skills in suicidal psychiatric patients. J. Consult. Clin. Psychol. 1987, 55, 49-54.

46. Williams, J. M. G. Suicide and Attempted Suicide: Understanding the cry of pain.; Penguin: London, 2001.

47. Tucker, R. P.; O'Connor, R. C.; Wingate, L. R. An Investigation of the Relationship Between Rumination Styles, Hope, and Suicide Ideation Through the Lens of the Integrated MotivationalVolitional Model of Suicidal Behavior. Arch. Suicide Res. 2016, 20, 553-566.

48. O'Connor, R. C.; Smyth, R.; Ferguson, E.; Ryan, C.; Williams, J. M. G. Psychological processes and repeat suicidal behavior: A four-year prospective study. J. Consult. Clin. Psychol. 2013, 81, 1137-1143.

49. O'Connor, R. C.; Rasmussen, S.; Hawton, K. Distinguishing adolescents who think about self-harm from those who engage in self-harm. Br. J. Psychiatry 2012, 200, 330-335.

50. Lubin, G.; Werbeloff, N.; Halperin, D.; Shmushkevitch, M.; Weiser, M.; Knobler, H. Y. Decrease in suicide rates after a change of policy reducing access to firearms in adolescents: a naturalistic epidemiological study. Suicide Life. Threat. Behav. 2010, 40, 421-424.

51. Mann, J. J.; Apter, A.; Bertolote, J.; Beautrais, A.; Currier, D.; Haas, A.; Hegerl, U.; Lonnqvist, J.; Malone, K.; Marusic, A.; Mehlum, L.; Patton, G.; Phillips, M.; Rutz, W.; Rihmer, Z.; Schmidtke, A.; Shaffer, D.; Silverman, M.; Takahashi, Y.; Varnik, A.; Wasserman, D.; Yip, P.; Hendin, H. Suicide Prevention Strategies: A Systematic Review. JAMA 2005, 294, 2064.

52. de Beurs, D.; Kirtley, O.; Kerkhof, A.; Portzky, G.; O'Connor, R. C. The Role of Mobile Phone Technology in Understanding and Preventing Suicidal Behavior. Crisis 2015, 36, 79-82.

53. Bagozzi, R. P.; Yi, Y. Specification, evaluation, and interpretation of structural equation models. J. Acad. Mark. Sci. 2012, 40, 8-34.

54. Borsboom, D.; Cramer, A. O. Network analysis: an integrative approach to the structure of psychopathology. Annu.Rev.Clin.Psychol. 2013, 9, 91-121.

55. Schmittmann, V. D.; Cramer, A. O. J.; Waldorp, L. J.; Epskamp, S.; Kievit, R. A.; Borsboom, D. Deconstructing the construct: A network perspective on psychological phenomena. New Ideas Psychol. 2013, 31, 43-53.

56. Isvoranu, A.-M.; van Borkulo, C. D.; Boyette, L.-L.; Wigman, J. T. W.; Vinkers, C. H.; Borsboom, D.; Group Investigators A Network Approach to Psychosis: Pathways Between Childhood Trauma and Psychotic Symptoms. Schizophr. Bull. 2017, 43, 187-196.

57. Cramer, A. O. J.; van der Sluis, S.; Noordhof, A.; Wichers, M.; Geschwind, N.; Aggen, S. H.; Kendler, K. S.; Borsboom, D. Dimensions of Normal Personality as Networks in Search of Equilibrium: You Can't Like Parties if You Don't Like People. Eur. J. Pers. 2012, 26, 414-431.

58. van Borkulo, C.; Boschloo, L.; Borsboom, D.; Penninx, B. W. J. H.; Waldorp, L. J.; Schoevers, R. A. Association of Symptom Network Structure With the Course of longitudinal Depression. JAMA psychiatry 2015, 72, 1219-1226.

59. American Psychiatric Association Diagnostic and statistical manual of mental disorder; 5th ed.; Washington, DC, 2013.

60. Fried, E. I.; Epskamp, S.; Nesse, R. M.; Tuerlinckx, F.; Borsboom, D. What are "good" depression symptoms? Comparing the centrality of DSM and non-DSM symptoms of depression in a network analysis. J. Affect. Disord. 2016, 189, 314-320.

61. de Beurs, D. P.; van Borkulo, C. D.; O'Connor, R. C. Association of suicidal symptoms and repeat suicidal behaviour within hospital treated suicide attempters. Br. J. Psychiatry Open, Accept. Publ. 2017.

62. Brooke, J. SUS: A quick and dirty usability scale. Usebility Eval. Ind. 1996, 189, $4-7$.

63. Bangor, A.; Kortum, P. T.; Miller, J. T. An Empirical Evaluation of the System Usability Scale. Int. J. Hum. Comput. Interact. 2008, 24, 574-594.

64. Mishara, B. L.; Weisstub, D. N. Ethical and legal issues in suicide research. Int. J. Law Psychiatry 2005, $28,23-41$. 